\title{
The Efficacy of Zinc(II) lons for Bacteria, Viruses, and Cancers Vaccines
}

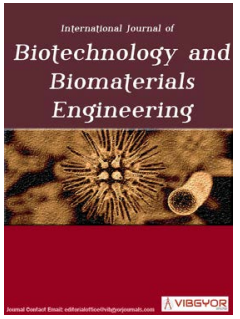

\section{Tsuneo Ishida*}

Life and Environment Science Research Division, Japan

\begin{abstract}
Zinc-dependent autolysin amidase induced bacterial vaccine may construct to be designed for AmiA, AmiB, and AmiC against Escherichia coli, and LytA against Streptococcus pneumoniae. The zinc-dependent autolysin endopeptidase induced vaccine may be considered for $D$, D-endopeptidase regulation against Gram-negative Vibrio cholerae. The zinc-dependent autolysin carboxypeptidase B1 induced vaccine also appears to be effective for TBVs against malaria eradication. The anti-bacterial vaccine activity of released $\mathrm{Zn}^{2+}$ ion occurs by zincmediated autolysin induced bacteriolysis.

The ability to create ZFN vaccine that can prevent and eliminate persistent viral infections is a long way from being realized. The efficacies by available LAV, live-attenuated rotavirus, influenza, and varicella zoster vaccines are strong incentive to redouble efforts to improve the safety characteristics of this type of vaccine. Vaccines for numerous infectious diseases have been developed using whole inactivated virions. ZOTENs prevent HSV-2 infection and disease with providing a platform for virus capture and presentation of neutralized virions. The antiviral vaccine activity may arise by zinc-mediated adapted immunity, viral apoptosis and death.

ZNF165 is novel cancer testis antigen capable of eliciting humoral immune response. ZNF165 induced HCC vaccine could be achieved as involved in tumor biology. ZMCs cancer vaccines have small molecular metal ion chelators and can be developed as the cancer therapeutics. MMP-2 may play a key role in angiogenesis and tumor growth that the vaccine based on chicken homologous MMP- 2 as a model antigen could induce both protective and therapeutic antitumor immunity. The anti-cancer vaccine activity may result by inhibition of tumor development, subsequently arising in the increase of cell-mediated immunity, and tumorous necrosis cell death. Hence, these novel targeted cancer therapeutics will be applied to cancer or tumor vaccine in future.
\end{abstract}

\section{Keywords}

Zinc-binding PGN autolysin, ZFN, Virus vaccine, ZOTEN, MMP, Cancer or tumor vaccine

\section{Abbreviations}

APC: Antigen Presenting Cell; CPBAs1: Carboxypeptidase B of Anopheles Gambiae Proteins; DCs: Dendritic Cells; EMT: Mesenchymal Transition; GAS: Group A Streptococcus, HCC: Hepatocellular

*Corresponding author: Dr. Sci. Tsuneo Ishida, Life and Environment Science Research Division, 2-3-6, Saido, Midori-Ku, Saitama-Shi, Saitama-Ken, 336-0907, Japan

Accepted: April 23, 2019; Published: April 25, 2019

Copyright: ( 2019 Ishida T. This is an open-access article distributed under the terms of the Creative Commons Attribution License, which permits unrestricted use, distribution, and reproduction in any medium, provided the original author and source are credited.

Ishida. Int J Biotechnol Biomater Eng 2019, 1:003 
Carcinoma; HIV: Human Immunodeficiency Virus; hMPV: Human Metapneumovirus; LAIV: Live Attenuated Influenza Vaccine; LAV: Live Attenuated Virus; MIBR: Most Probable Immunoprotective B-cell Epitope Regions; MMP: Matrix Metalloproteinase; PGN: Peptidoglycan; PSP: Plasmid Stabilization Protein; PRRSV: Porcine Reproductive and Respiratory Syndrome Virus; RSV: Respiratory Syncytial Virus; TALENs: Transcription Activator-like Effector Nucleases; TBV: Transmission-Blocking Vaccine; Th: T Helper; TG: Transglycosylase; TP: Transpeptidase; ZBL: Zinc Binding Lipoprotein; ZF: Zinc Finger; ZFAs: Zinc Finger Arrays; ZFN: Zinc Finger Nuclease; ZNF: Zinc-Finger Protein; ZMCs: Zinc Metallochaperones; ZOTENs: ZnO Tetrapod Nanoparticles

\section{Introduction}

Hydrolysis and degradation activities of $\mathrm{Zn}^{2+}$ ions have been investigated for bacteriolyses of bacterial cell walls, viral protein and RNA degradations, and regulation of cancer/tumor cell growth [1]. $\mathrm{Zn}^{2+}$ ions-mediated hydrolytic and degradative functions for bacteria are due to bacteriolyses and destructions of bacterial cell walls by the inhibitions of PGN elongation owing to activated peptidoglycan (PGN) autolysins. $\mathrm{Zn}^{2+}$ ions-mediated degradative function for viruses is viral protein hydrolysis, viral mRNA degradation, and bacteriophage viral-endolysins, by that $\mathrm{Zn}^{2+}$ ion inhibits HIV-1 by recruiting the $5^{\prime}$ and $3^{\prime}$ mRNA degradation, specifically promotes the degradation of multiply spliced HIV-1 m-RNAs. Zinc selectively and markedly down-regulated nonstructural protein levels by increasing protein degradation. Furthermore, $\mathrm{Zn}^{2+}$-mediated hydrolyzing and degrading function for cancer cell is related to protea some and autophage that leads to cancer and tumor cell death, $\mathrm{Zn}^{2+}$ ions inhibit malignant tumor proliferation and metastasis, and zinc complex and zinc chelation that have important roles for anticancer/tumor apoptotic death. Thus, these hydrolysis and degradation methods have being now noteworthy under the hydrolase enzymic development for bactericidal, virucidal, and cancerous cell death [1].

Zinc can inhibit apoptosis induced by both chemical and death-receptor agonists. Apoptotic effects of zinc because zinc is reported to both induce apoptosis in some cancers and to protect other cancer cells against apoptosis induced by other factors. Zinc played a key role in the regulation of epithelial to mesenchymal transition (EMT) and metastatic behaviors that zinc-induced EMT increases the intracellular superoxide anion and induces EMT phenotypes in lung cancer cells by up-regulating of EMT markers and down-regulating of E-cadher in protein. $\mathrm{Zn}^{2+}$ ions promote hydrolysis of RNA and protein. Zinc ion-dependent hydrolysis of RNA cleaves RNA that $\mathrm{Zn}^{2+}$-promoted cleavage was observed to be considerably more sensitive to the secondary structure of the chain [2].

The progress in the vaccine development is closely related with the progress in immunology and avoiding disease from the vaccine itself. The immunogenicity of zinc finger nuclease (ZFN) vaccines can be controlled by temporal and spatial regulation of zinc finger (ZF) expression to balance viral protein expression with the ability of the ZFNs to eliminate all replication-competent viral DNA [3]. By controlling viral replication temporally or spatially, a strong, natural immune response can be elicited before the virus is eliminated that these may be particularly useful approaches for designing vaccines against persistent or latent viruses, as ZFNs and miRNAs lead to the elimination of all viral DNA or RNA, thus preventing chronic infection [3].

Streptococcus pyogenes, known as group A streptococcus (GAS), is a human pathogen that causes a variety of clinical manifestations, and disease prevention is hampered by lack of a human GAS vaccine, in which an immunization of mice with the extracellular component of the zinc importer confers protection against systemic GAS challenge and a similar struggle for zinc may occur during other streptococcal infection [4]. Thus, it is of importance that availability of these hydrolysis, degradation, and surface death-receptor is applied to $\mathrm{Zn}$-mediated infectious vaccine and vaccination. Zinc-mediated infectious vaccine is unknown only the beginning with because the vaccinology is not yet established.

In this mini-review, firstly, the effect of zinc(II) on zinc-binding peptidoglycan (PGN) autolysin induced enhancement of anti-bacterial vaccine has been investigated. Secondly, zinc-mediated antiviruses vaccine and zinc-dependent anti-cancer or anti-tumor vaccine have been investigated. Lastly, the efficacy of $\mathrm{Zn}^{2+}$ ions for zinc-mediated infectious and cancerous vaccines has been discussed. 
Zinc-Mediated Vaccine Development against Bacteria; Zinc-dependent Autolysins Amidase/Endopeptidase/Carboxypeptidase In-

\section{duced Bacterial Vaccine Inactivation}

In the molecular structure of $S$. aureus PGN cell wall, there are action sites of transglycosylase (TG) and transpeptidase (TP) mainly on thick PGN layer. The TG is the synthetic enzymes of $\mathrm{N}$-acetylglucosamidase cleavage between NAG ( $\mathrm{N}$-acetylglucosamine) and NAM ( $\mathrm{N}$-acetylmuramic acid), and $\mathrm{N}$-acetylmuramidase cleavage between NAM and NAG on glycan chain. The TP is the synthetic enzyme cleavage between Glycine and $D$-alanine on PGN crosslinking. The other, there are PGN autolysins of $\mathrm{N}$-acetylmuramyl-L-alanine amidase cleavage, $D D$-endopeptidases cleavages between Glycine and Glycine on pentaglycine (Gly) 5 , and in addition, lysostaphin cleavage between Glycine and Glycine on PGN cross-linking [5]. PGN autolysins are bacterial autolysins such as amidase, endopeptidase, and carboxypeptidase, play bacteriolytic destructions of bacterial cell walls due to inhibition of PGN elongation. The zinc-binding autolysin induced vaccine design is considered against bacteria. Autolysin, zinc binding lipoprotein (ZBL), and plasmid stabilization protein (PSP) were already identified as pneumococcal surface proteins able to elicit protection against S. pneumoniae serotype $19 \mathrm{~F}$ and their most probable immunoprotective B-cell epitope regions (MIBRs) were determined that the chimerical antigen composed of conserved MIBRs is a suitable vaccine candidate, which can elicit protection against common pneumococcal serotypes [6]. Zinc dependent autolysin amidase induced bacterial vaccine may construct to be designed for AmiA, $\mathrm{AmiB}$, and AmiC against Escherichia coli [7], and LytA against Streptococcus pneumoniae [8]. Zinc dependent autolysin endopeptidase induced bacterial vaccine may be considered for $D$, $\mathrm{D}$-endopeptidase regulation against Gram-negative Vibrio cholerae [9]. Zinc-dependent autolysin carboxypeptidase B1 induced bacterial vaccine also appears to be effective for transmission-blocking vaccines (TBVs) against malaria eradication that carboxypeptidase B of Anopheles gambiae proteins (CPBAs1) could be introduced as a TBV candidate in regions where $A$. stephensi is the main malaria vector [10]. Thus, $\mathrm{Zn}^{2+}$ ion induced anti-bacterial vaccine activity occurs by bacteriolysis of bacterial cell walls due to activated bacterial PGN autolysins through $\mathrm{Zn}^{2+}$ ions induction.

\section{Zinc-Mediated Vaccine Development against Viruses}

The ability to create a zinc finger nuclease (ZFN) vaccine that can prevent and eliminate persistent viral infections is a long way from being realized, in which the efficacies by available live, attenuated virus (LAV), live-attenuated rotavirus, influenza, and varicella zoster vaccines are strong incentive to redouble efforts to improve the safety characteristics of this type of vaccine [3]. Vaccines for numerous infectious diseases have been developed using whole inactivated virions.

The inhibition of zinc binding activity of human metapneumovirus (hMPV) M2-1 protein can lead to the development of novel, live attenuated vaccines as well as antiviral drugs for pneumoviruses [11]. Identified hMPV as an important causative agent of acute respiratory diseases in infants is studied, the elderly, and there are currently no licensed therapeutics or vaccines against hMPV, in which new approaches for immunization and therapy are obtained against human metapneumovirus [12].

The $\mathrm{CCHH}$ zinc finger motif provides a critical determinant for virulence in mouse and mutations in the $\mathrm{CCHH}$ motif yield potential vaccine candidates for the development of live species-specific attenuated influenza virus vaccines [13]. The live attenuated influenza vaccines (LAIVs) are attracting attention as an effective strategy due to several advantages over inactivated vaccines [14]. Zinc finger reactive compounds also inactivate retroviruses by targeting the zinc finger motif in their nucleocapsid proteins. These compounds are effective against respiratory syncytial virus (RSV) that AT-2-inactivated RSV vaccine may not be enough to produce a highly-efficacious inactivated virus vaccine which does not lead to an atypical disease [15].

Zinc sulfate has no effect in level of immunity among elderly and zinc may stimulate antibody response in the non-responders to recombinant hepatitis $B$ vaccine. The effect of zinc sulfate on seroconversion after a simple method vaccination had been identified that accelerated HB vaccination can shorten duration of immunization of this clinical trial for showing its effectiveness [16]. A human papillomavirus type 16 E7 DNA vaccine indicated a significantly stronger E7-specific cytotoxic T-lym- 
phocyte induction and better antitumor protection [17].

Zinc finger nucleases (ZFNs) can specifically and efficiently excise HIV-1 proviral DNA from latently infected human $T$ cells, the resulting from HIV-1infected human primary $T$ cells and latently infected $T$ cells treated with the inducible ZFNs validated that proviral DNA can be excised [18]. The regulated ZFNs in the presence of HIV-1 Tat may provide a safer and novel genome-editing technology for eradicating HIV-1 proviral DNA from infected host cells. ZFNs and transcription activator-like effector nucleases (TALENs) bear sequence-specific DNAbinding modules that recognize HIV DNA sequences [19]. Therapeutic-potential of ZFNs and zinc finger arrays (ZFAs) is confirmed with unique specificity to HSV-2 the genome which ZFNs with specificity to HSV-2 genomic DNA are precursors of novel host genome expressed HSV-2 gene-therapeutics or vaccines [20].

Higher levels of dietary zinc oxide $(\mathrm{ZnO})$ levels could not provide enhanced protection against porcine reproductive and respiratory syndrome virus (PRRSV) vaccine and infection that have the potential to stimulate or modulate systemic immune responses after vaccination [21]. In the synthesized $\mathrm{ZnO}$ tetrapod nanoparticles (ZOTENs), ZOTENs prevent HSV-2 infection and disease that ZOTEN provides a platform for virus capture and presentation of neutralized virions to mucosal antigen presenting cell (APCs), for initiating and boosting adaptive immunity which provides the novel evidence for the protective efficacy of an intravaginal microbicide/vaccine or microbivac platform against primary and secondary female genital herpes infection [22]. These anti-viruses vaccines mechanism is indicated that the vaccine activity of released $\mathrm{Zn}^{2+}$ ions from zinc solutions and $\mathrm{ZnO}$ NPs, may occur by $\mathrm{Zn}^{2+}$ ion-induced adapted immunity, viral apoptosis and death.

\section{Zinc-Mediated Vaccine Development against Tumor Cell}

Therapeutic cancer vaccines are designed to generate a targeted, immune-mediated antitumor response, but a tough challenge for the majorities of tumor vaccines is the self-nature of tumor antigens that the antigen formulation is essential for a vaccine to be effective [23]. More recently, the concept of vaccination has been developed into a potentially novel strategy to treat and prevent cancer formation, progression, and spread that with more anti-cancer vaccines currently in development, they can eventually become routine tools used in the treatment and prevention of cancer in the future [24]. Next generation cancer vaccines are combining immune check point inhibitors to overcome the immune-suppressive microenvironment and personalized cancer vaccines for directing the host immune system against the chosen antigens [25].

Zn homeostasis impacts maturation of dendritic cells (DCs) that are important in shaping $T$ cell response, in which $\mathrm{Zn}$ shapes the tolerogenic potential of DCs and promotes Tregs in regulatory $T$ cell (Treg)-Th ( $T$ helper)17 balance during fungal infection [26]. These DCs are required for anti-cancer vaccine with genetic modification and combination with other strategies including adoptive T-cell transfer [27]. Hierarchical $\mathrm{Cu}$ - and $\mathrm{Zn}$-buds dressing $\gamma$-AlOOH mesostrands, which are oriented in randomly wrinkled matrice, are suitable platforms as novel adjuvants for immunotherapy that alum is only licensed adjuvant by USA drug administration used in many human vaccines, and alum hardly induces T helper 1 (Th1) immune responses that are required for anti-tumor immunity [28].

Zinc-finger protein ZNF165 is a novel cancertestis antigen capable of eliciting humoral immune response and be involved in tumor biology that ZNF165 induced hepatocellular carcinoma (HCC) vaccine could be achieved [29]. A new class of mutant p53 called Zinc metallochaperones (ZMCs) is developed as the cancer therapeutics and cancer vaccines which ZMCs are small molecular metal ion chelators that bind zinc and other divalent metal ions strong enough to remove zinc from serum albumin, but weak enough to donate it to mutant p53 [30].

In particular, MMP-2 including matrix metalloproteinase (MMP) family may play a key role in angiogenesis and tumor growth that the vaccine based on chicken homologous MMP-2 as a model antigen could induce both protective and therapeutic antitumor immunity [31]. A vaccine strategy for cancer therapy may be provided through the induced autoimmune response against MMP-2 by the immunization with the single xenogeneic homologous MMP-2 gene, in which MMP-2 induced 
active immunogene therapy with cancer vaccine was apparently inhibited by the vaccination with MMP-2 [31]. MMPs11 also as a novel target antigen for cancer immunotherapy are zinc-dependent endopeptidases with matrix degradation, tissue remodeling, inflammation, and tumor metastasis, in which the identification of MMPs11 as a novel broadly expressed tumor associated antigen as target candidate for cancer immunotherapy and cancer vaccine [32]. These cancer vaccine mechanism shows that released $\mathrm{Zn}^{2+}$ ions induced anti-cancer vaccine activity could result by inhibiting in vivo tumor development using re-challenged LLC cells in combination with an autolysin tumor tissue which may subsequently result in an increase in the degree of secretion of cytokines such as tumor necrosis factor (TNF $\alpha$ ) and interferon (IFNY), in the increase in cell-mediated immunity, and tumorous necrosis and tumor cell death. Thus, these novel targeted cancer therapeutics will be applied to cancer or tumor vaccine in future.

\section{Conclusions}

(1) Zinc-dependent autolysin amidase induced bacterial vaccine may construct to be designed for AmiA, AmiB, and AmiC against Escherichia coli, and LytA against Streptococcus pneumoniae. Zinc-dependent autolysin endopeptidase induced vaccine may be considered for $D$, D-endopeptidase regulation against Gram-negative Vibrio cholerae. Zinc-dependent autolysin carboxy-peptidase B1 induced vaccine also appears to be effective for TBVs against malaria eradication. The anti-bacterial vaccine activity occurs by zinc-mediated autolysin induced bacteriolysis of bacterial cell walls.

(2) The ability to create a ZFN vaccine that can prevent and eliminate persistent viral infections is a long way from being realized, in which the efficacies by available LAV, live-attenuated rotavirus, influenza, and varicella zoster vaccines are strong incentive to redouble efforts to improve the safety characteristics of this type of vaccine. Identified hMPV as an important causative agent of acute respiratory diseases in infants is studied, the elderly, and there are currently no licensed therapeutics or vaccines against hMPV, in which new approaches for immunization and therapy are obtained against human metapneumovirus.

Higher levels of dietary $\mathrm{ZnO}$ levels could not provide enhanced protection against PRRSV vaccine and infection that have the potential to stimulate or modulate systemic immune responses after vaccination. The synthesized ZOTENs prevent HSV-2 infection and disease. ZOTEN provides the novel evidence for the protective efficacy of an intravaginal microbicide/vaccine or microbivac platform against primary and secondary female genital herpes infection. The anti-viral vaccine activity may arise by zinc-mediated adapted immunity, viral apoptosis and death.

(3) Zinc-finger protein ZNF165 is a novel cancertestis antigen capable of eliciting humoral immune response and ZNF165 induced HCC vaccine could be involved and achieved in tumor biology. ZMCs are developed as the cancer therapeutics and cancer vaccines which ZMCs are small molecular metal ion chelators that bind zinc and other divalent metal ions strong enough to remove zinc from serum albumin, but weak enough to donate it to mutant p53. MMP-2 may play a key role in angiogenesis and tumor growth that the vaccine based on chicken homologous MMP-2 as a model antigen could induce both protective and therapeutic antitumor immunity. Hence, a vaccine strategy for cancer therapy may be provided through the induced autoimmune response against MMP-2 by the immunization with the single xenogeneic homologous MMP-2 gene. MMPs11 also as a novel target antigen for cancer immunotherapy are zinc-dependent endopeptidases with matrix degradation, tissue remodeling, inflammation, and tumor metastasis. Released $\mathrm{Zn}^{2+}$ ions induced anticancer vaccine activity could result by inhibiting tumor development, an autolysin tumor tissue which may subsequently result in the cell-mediated immunity, tumorous necrosis and tumor cell death. Thus, these novel targeted cancer therapeutics will be applied to cancer or tumor vaccine in future.

Finally, in the development of these bacteria, viruses, and cancer vaccines, it is of importance that exciting vaccine research on zinc-mediated vaccinology may become essential for pursuing molecular biology.

\section{Conflicts of Interest}

The author declares no conflict of interest.

\section{Funding}

There was no funding received for this study. 


\section{References}

1. T Ishida (2018) Hydrolytic and degradative activities of $\mathrm{Zn}^{2+}$ ions for bacterial cell walls, viral RNA degradation, and regulation of tumorous cell growth. ARC Journal of Immunology and Vaccines 3: 1-6.

2. IZagorowska, S Kuusela, H Lonnberg (1998) Metal iondependent hydrolysis of RNA phosphodiester bonds within hairpin loops. A comparative kinetic study on chimeric ribo/2'-O-methylribo oligonucledotides. Nucleic Acids Res 26: 3392-3396.

3. AS Lauring, JO Jones, R Andino (2010) Rationalizing the development of live attenuated virus vaccines. 28: 573-579.

4. Makthal N, Nguyen K, Do H, Gavagan M, Chandrangsu $P$, et al. (2017) A critical role of zinc importer AdcABC in group A Steptococcus-host interactions during infection and its implications for vaccine development. EBioMedicine 21: 131-141.

5. Tsuneo Ishida (2016) Bacteriolyses of $\mathrm{Cu}^{2+}$ solution on bacterial cell walls/cell membrane and DNA base pairing damages. Biomedical Research on Trace Elements 27: 151-161.

6. Shirin Tarahomjoo (2016) Serotype independent vaccine design against Streptococcus pneumoniae based on B-cell epitopes of autolysin, zinc binding lipoprotein and plasmid stabilization protein. American Journal of Medical and Biological Research 4: 84-89.

7. JD Lenz, EA Stohl, RM Robertson, KT Hackett, K Fisher, et al. (2016) Amidase activity of AmiC controls cell separation and stem peptide release and is enhanced by NIpD in Neisseria gonorrhoeae. Journal of Biological Chemistry 291: 10916-10933.

8. P Mellroth, T Sandalova, A Kikhney, F Vilaplana, D Hesek, et al. (2014) Structural and functional insights into peptidoglycan access for the lytic amidase LytA of Streptococcus pneumoniae. MBio 5: e01120-13.

9. SG Murphy, L Alvatez, MC Adams, S Liu, JS Chappie, et al. (2019) Endopeptidase regulation as a novel function of the Zur-Dependent zinc starvation response. MBio 10: e02620-18.

10.A Raz, ND Dinparast, S Zakeri (2013) Molecular characterization of the carboxypeptidase B1 of Anopheles Stephensi and its evaluation as a target for transmission-blocking vaccines. Infect Immun 81: 2206-2216.

11. H Cai, Y Zhang, Y Ma, J Sun, X Liang, et al. (2015) Zinc binding activity of human metapneumovirus M2-1 protein is indispensable for viral replication and pathogenesis in vivo. J Virol 89: 6391-6405.
12.Sherry C Wen, John V Williams (2015) New approaches for immunization and therapy against human metapneumovirus. Clin Vaccine Immunol 22: 858-866.

13.E Ka-Wai Hui, DF Smee, Min-Hui Wong, DP Nayak (2006) Mutations in influenza virus $\mathrm{M} 1 \mathrm{CCHH}$, the putative zinc finger motif, cause attenuation in mice and protect mice against lethal influenza virus infection. J Virol 80: 5697-5707.

14.Yo Han Jang, Baik-Lin Seong (2012) Principles underlying rational design of live attenuated influenza vaccines. Clin Exp Vaccine Res 1: 35-49.

15.MS Boukhvalova, GA Prince, JC Blanco (2010) Inactivation of respiratory syncytial virus by zinc finger reactive compounds. Virol J 7: 20.

16.M Afsharian, S Vaziri, AR Janbakhsh, B Sayad, F Mansouri, et al. (2011) The effect of zinc sulfate on immunologic response to recombinant hepatitis $B$ vaccine in elderly: Zinc sulfate and immunologic response to recombinant hepatitis $B$ vaccine. Hepat Mon 11: 32-35.

17.W Shi, P Bu, J Liu, A Polack, S Fisher, et al. (1999) Human papillomavirus type 16 E7 DNA vaccine: Mutation in the open reading frame of E7 enhances specific cytotoxic T-lymphocyte induction and antitumor activity. J Virol 73: 7877-7881.

18. H Ji, P Lu, B Liu, X Qu, Y Wang, et al. (2018) Zinc-finger nucleases induced by HIV-1 tat excise HIV-1 from the host genome in infected and latently infected cells. Mol Ther Nucleic Acids 12: 67-74.

19.C Liang, MA Wainberg, AT Das, B Berkhout (2016) CRISPR/Cas9: A double-edged sword when used to combat HIV infection. Retrovirology 13: 37-41.

20.Misaki Wayengera (2011) Identity of zinc finger nucleases with specificity to herpes simplex virus type II genomic DNA: Novel HSV-2 vaccine/therapy precursors. Theor Biol Med Model 8: 1-13.

21.W Chai, Z Wang, P Janczyk, S Twardziok, U Blohm, et al. (2014) Elevated dietary zinc oxide levels do not have a substantial effect on porcine reproductive and respiratory syndrome virus (PRRSV) vaccination and infection. Virol J 11: 1-5.

22.TE Antoine, SR Hadigal, AM Yakoub, YK Mishra, Bhattacharya P, et al. (2016) Intravaginal zinc oxide tetrapod nanoparticles as novel immunoprotective agents against genital herpes. J Immunol 196: 45664575.

23. Matteo Lazzeroni, Davide Serrano (2012) Potential use of vaccines in the primary prevention of breast cancer in high-risk patients. Breast Care (Basel) 7: 281-287. 
24.Justin KH Liu (2014) Anti-cancer vaccines - A one-hit wonder? Yale J Biol Med 87: 481-489.

25.Angelika Terbuch, Juanita Lopez (2018) Next Generation Cancer Vaccines - Make it Personal! Vaccines. 6: Doi: 10.3390, PP: 1-17.

26.MM George, KS Vignesh, JAL Figueroa, Caruso JA, Deepe GS Jr (2016) Zinc induces dendritic cell tolerogenic phenotype and skews regulatory $T$ cellTh17 balance. J Immunol 197: 1864-1876.

27.ME Turnis, CM Rooney (2010) Enhancement of dendritic cells as vaccines for cancer. Immunotherapy 2: 847-862.

28.X Li, MA Shenashen, X Wang, A Ito, A Taniguchi, et al. (2017) Hierarchically porous, and $\mathrm{Cu}$ - and $\mathrm{Zn}$ containing $\mathrm{\gamma}$-AlOOH mesostrands as adjuvants for cancer immunotherapy. Scientific Reports 7: 1-10..
29.XY Dong, XA Yang, YD Wang, WF Chen (2004) Zincfinger protein ZNF165 is a novel cancer-testis antigen capable of eliciting antibody response in hepatocellular carcinoma patients. $\mathrm{Br} J$ Cancer 91: 1566-1570.

30.Samuel Kogan, Darren R Carpizo (2018) Zinc metallochaperones as mutant p53 reactivators: A new paradigm in cancer therapeutics. Cancers (Basel) 10: P 1-13.

31.JM Su, YQ Wei, L Tian, X Zhao, L Yang, et al. (2003) Active immunogene therapy of cancer with vaccine on the basis of chicken homologous matrix metalloproteinase-2. Cancer Res 63: 600-607.

32.D Peruzzi, F Mori, A Conforti, D Lazarrow, E De Rinaldis, et al. (2009) MMP11: A novel target antigen for cancer immunotherapy. Clin Cancer Res 15: 4104-4113. 